\title{
Broadband Wireless Communications
}

\author{
3G, 4G and Wireless LAN
}




\section{THE KLUWER INTERNATIONAL SERIES}

IN ENGINEERING AND COMPUTER SCIENCE 


\section{BROADBAND WIRELESS COMMUNICATIONS 3G, $4 G$ and Wireless LAN}

by

Jiangzhou WANG

University of Hong Kong

KLUWER ACADEMIC PUBLISHERS

NEW YORK, BOSTON, DORDRECHT, LONDON, MOSCOW 
eBook ISBN: $\quad$ 0-306-46999-5

Print ISBN: $\quad$ 0-7923-7391-X

(C2002 Kluwer Academic Publishers

New York, Boston, Dordrecht, London, Moscow

Print @2001 Kluwer Academic Publishers

All rights reserved

No part of this eBook may be reproduced or transmitted in any form or by any means, electronic, mechanical, recording, or otherwise, without written consent from the Publisher

Created in the United States of America

Visit Kluwer Online at:

http://kluweronline.com

and Kluwer's eBookstore at:

http://ebooks.kluweronline.com 
To

Leiping, April, Angela and Larry 


\section{TABLE OF CONTENTS}

Preface $x i$

Acknowledgements $\quad$ XV

Chapter 1: 3G Mobile Communications 1

1.1 Introduction 2

1.2 Multiple Access 4

$\begin{array}{lll}1.3 & \text { Channel Coding } & 7\end{array}$

1.4 Rate Matching 12

1.5 Modulation and Spreading 13

1.6 Physical Layer Procedures 24

1.7 Transmit Diversity 28

Chapter 2: Wideband CDMA $\quad 34$

2.1 Overview 35

2.2 System Models 36

2.3 Channel Estimation Errors 39

2.4 Numerical Computation 49

2.5 Envelope Variations 54

2.6 Summary 61

Chapter 3: Multicode CDMA 64

3.1 Introduction 65

3.2 Multicode CDMA Models 66

3.3 Analysis of MCI Cancellation 73

3.4 System Performance 86

3.5 Numerical Results $\quad 87$

3.6 Conclusions 91

Chapter 4: $\quad$ Advanced Tracking Loop 94

4.1 Overview 95

4.2 System Models $\quad 99$

4.3 Performance Analysis 102

4.4 Bandlimited Received Signal 109

4.5 Examples 111

4.6 Effect of Multipath Signals 115

4.7 Discussions 117 
viii Broadband Wireless Communications

Chapter 5: CDMA Overlay

5.1 Overview 123

5.2 System Model 127

5.3 System Performance 131

5.4 Numerical Results 137

$\begin{array}{lll}5.5 & \text { Conclusions } & 143\end{array}$

Chapter 6: Adaptive Filtering in CDMA Overlay 147

6.1 Introduction 148

6.2 Filter Coefficients 148

6.3 Signal to Noise Ratio 157

6.4 LMS Filtering 161

6.5 Numerical and Simulation Results 163

Chapter 7: Open Loop Power Control in CDMA Overlay 175

$\begin{array}{lll}7.1 & \text { Overview } & 176\end{array}$

7.2 Downlink System Description 182

7.3 Power Control Error 191

7.4 Numerical Results 198

$\begin{array}{lll}7.5 & \text { Conclusions } & 202\end{array}$

Chapter 8: Closed Loop Power Control 206

8.1 Introduction 207

8.2 System Model 208

8.3 Power Control Model 211

8.4 System Performance 219

8.5 Numerical Results 220

8.6 Conclusions 223

Chapter 9: Uplink Multicarrier DS/SFH-CDMA 225

9.1 Literature Review 226

9.2 Channel and Interference Models 228

9.3 System Model 230

9.4 Performance Analysis 234

9.5 Numerical Results 240

9.6 Conclusions 244

Appendix 9A 245

Appendix 9B 248

Chapter 10: Downlink Multicarrier DS/SFH-CDMA 252

10.1 Introduction 253

10.2 Systems Model 253

10.3 Performance Evaluation 254

10.4 Numerical Results 260 
10.5 Conclusions 265

Chapter 11: Wireless Frequency Hopping 266

$\begin{array}{lll}11.1 \text { Overview } & 267\end{array}$

$\begin{array}{lll}11.2 & \text { System Models } & 269\end{array}$

11.3 Statistical Description 274

11.4 Bit Error Rate (BER) 279

11.5 Numerical Results 285

11.6 Conclusions 289

Chapter 12: A Novel FHMA Receiver 290

12.1 Introduction 291

12.2 System Models 292

12.3 Analysis 295

12.4 Numerical Results $\quad 305$

$\begin{array}{lll}12.5 & \text { Conclusions } & 309\end{array}$

Chapter 13: 4G Mobile Communications $\quad 310$

13.1 Market Trend 311

13.2 Key Technologies 315

Appendix 13 OFDM+CDMA 318

$\begin{array}{ll}\text { Abbreviations } & 327\end{array}$

Index 332

The Author 337 


\section{Preface}

The broadband wireless communications field is growing at an explosive rate, stimulated by a host of important emerging applications ranging from $3 \mathrm{G}, 4 \mathrm{G}$ and wireless LAN. Wideband CDMA and CDMA2000 will be used for 3G. OFDM+CDMA might be a good choice for 4G, CDMA overlay will possibly be used for new-generation broadband wireless LAN. For system planners and designers, the projections of rapidly escalating demand for such wireless services present major challenges and meeting these challenges will require sustained technical innovation on many fronts.

The text of this book has been developed through years of research by the author and his graduate students at the University of Hong Kong. The aim of this book is to provide a R\&D perspective on the field of broadband wireless communications by describing the recent research developments in this area and also by identifying key directions in which further research is needed. As a background, I presume that the reader has a thorough understanding of digital communications and spread spectrum/CDMA.

The book is arranged into 13 chapters. In chapter 1, some key specifications of 3G WCDMA are described and discussed. These techniques include channel coding, rate matching, modulation and spreading, power control, cell search, transmit diversity, soft-handoff, and so son.

In Chapter 2, the coherent RAKE reception of Wideband CDMA signals with complex spreading is considered. A dedicated pilot channel, which is separate from data channels, is used for the purpose of channel estimation. Based on a digital implementation, the coherent demodulation scheme is presented. Pilot channel estimation error due to multiple access and multipath interference is studied. System performance is evaluated by means of bit error rate. The analysis shows that the error of channel estimation significantly degrades system performance and can be effectively suppressed by low pass filters (LPFs). A discussion on the envelope variation of complex spread signals is also included, which illustrates that the complex spread signal has more stable envelope than the dual-channel spread signal.

Multicode CDMA is a new transmission scheme for flexible and highspeed data communications. The basic idea of multicode CDMA is to assign multiple channelization codes to any given user. If these channel codes are orthogonal, the self-interference among them can be eliminated in an AWGN channel. However, in a multipath environment, these intra-user signals from different delay paths no longer maintain orthogonality and thus cause 


\section{xii Broadband Wireless Communications}

interference, i.e. multicode interference (MCI), to each other. In high-speed data networks, where the number of users is much less than in the voice networks, the MCI may represent a large portion of the total interference and has great impact on the system performance. In Chapter 3, a complex spreading multicode wideband CDMA receiver with RAKE structure and MCI cancellation is studied. By pilot aided channel estimation, the MCI associated with the reference user is regenerated and subtracted from the received signal. A complete and consolidated theoretical analysis is presented to show that the system performance is significantly improved by the MCI cancellation.

In Chapter 4, half-sine and triangular chip waveforms are proposed for the locally generated early and late despreading codes in a coherent DLL for a single user in a DS/SS systems. Both ideal and band-limited received signals are investigated. These non-rectangular waveforms reduce the effective loop noise power with only minor effect on the DLL sensitivity.

Chapter $\mathbf{5}$ is concerned with a direct sequence code division multiple access (DS-CDMA) system sharing common spectrum with a narrowband waveform for wireless LAN applications. A suppression filter at a receiver is employed to reduce the narrowband interference. The average up-link bit error rate (BER) performance is investigated how to be influenced by various parameters. Chapter 6 presents the behavior of reflection coefficients of a stochastic gradient lattice (SGL) filter applied to a CDMA overlay system. Analytic expressions for coefficients for a two stage filter are derived with the presence of narrowband interference and additive white gaussian noise (AWGN). It is shown that the coefficients of the lattice filter exhibit separate tracking and convergent properties and that compared to a LMS filter, the lattice filter provides fast rate of convergence, while having good capability of narrowband interference suppression.

Adaptive power control has widely been used in DS/CDMA systems to overcome the so-called "near-far" problem. Chapter 7 studies the adaptive open-loop power control of a cellular CDMA system, which is overlaid in downlink by a narrowband signal. The effects of downlink power allocation schemes to power control error in the presence of narrowband interference are analyzed and numerically computed. In order to get a minimum power control error in the CDMA overlay situations, an optimum downlink power allocation scheme is used, which works well for wide range of signal to narrow-band interference ratio. In Chapter 8, we investigate the effect of power control error of a closed-loop power controlled CDMA system with imperfect power estimation. By use of a simplified log-linear power control model, the power control error of a strength-based power control algorithm is studied in the presence of narrowband interference.

In Chapter 9, uplink multicarrier direct-sequence/slow-frequencyhopping (MC DS/SFH) CDMA systems are proposed, in which multiple 
carriers are modulated by the same DS waveform and hopped in frequency according to a random hopping pattern. The receiver dehops the received signal with the same pattern, provides RAKE receivers for each carrier and combines the outputs with a maximal ratio combiner (MRC). The performance of the proposed system is investigated over a frequency selective Rayleigh fading channel and compared to that of the MC DSCDMA systems. This scheme is suitable for uplink transmission where BS can have more antennas. In Chapter 10, another type of the slow frequency hopping (SFH) technique to the multicarrier (MC) CDMA systems for downlink overlay situations. Using lower chip rate, which results in a narrower spectrum for each carrier and hopping the signal in frequency, the MC DS/SFH system achieves better performance than the MC DS CDMA system in most cases, especially when the bandwidth of the narrowband interference is narrower than one subchannel. This scheme is good at downlink where MS does not have multiple antennas.

Frequency hopping is an alternative technique for wireless mobile communications. Chapter $\mathbf{1 1}$ is concerned with the bit error rate (BER) performance of a cellular one-hop/symbol frequency hopping multiple access (FHMA) system operating through a multipath Rayleigh fading channel The multiple access and adjacent cell interference of the cellular FHMA system has been studied. In Chapter 12, a novel FH/MFSK receiver with additional partial correlators is proposed by means of side information for asynchronous frequency hopping multiple-acess (FHMA) systems in the presence of Rayleigh fading. Performance analysis is carried out and compared for both the novel and conventional receivers.

Finally, with both economic growth and technology development of the world, multimedia services with even higher data rate (i.e. 20Mbps) will be provided by 4G. In Chapter 13, the forecast of mobile communications and key techniques of $4 \mathrm{G}$ will be presented. 


\section{Acknowledgements}

Throughout my professional carrier, I have had the opportunity to work with and learn from the following people whom I would like to greatly acknowledge:

Professor Laurence B. Milstein University of California at San Diego, USA Professor Tung-Sang Ng University of Hong Kong

Professor Fumiyuki Adachi Tohoku University, Japan

Professor Marc Moeneclaey University of Ghent, Belgium

Dr. Mamoru Sawahashi $\quad$ NTT DoCoMo, Japan

Dr. Ker Zhang Synchronization Inc., USA

Dr. Zhengmao Li China Unicom

Professor Milstein introduced me to CDMA overlay during my postdoctoral research. Since then, I have published extensively in this area. Professor $\mathrm{Ng}$ has been giving me much encouragement and support in my professorial carrier. Professor Adachi selflessly introduced me to several key issues in wideband CDMA. Professor Moeneclaey effectively supervised my Ph.D. dissertation. Dr. Sawahashi helped me much with patience in the understanding of $3 \mathrm{G}$ standard during my visit to NTT DoCoMo. Dr. Zhang was a Group Leader when I worked in a US company. Dr. Li was my classmate during my early Ph.D. study.

During the past a few years, I have had pleasure to supervise a number of hardworking graduate students, without whom this book would have never appeared. I would like to express my gratitude to:

Chen Jiang

$\mathrm{Ai} \mathrm{Yu}$

Vicknarajah Prahatheesan

Thayaparan Subramaniam

Hu Huang

Xi Zhang
Jun Chen

Xiangyang Wang

Haidong $\mathrm{Wu}$

Yiqing Zhou

Bin Xia

This book is the product of funded research supported by NTT DoCoMo, Japan, the RGC of Hong Kong SAR Government and the University of Hong Kong. Their financial support is appreciated.

Finally, I wish to thank my wife, Leiping $\mathrm{Xu}$, and three children, April Pingyang, Angela Pingyu, Larry Pinglei for their encouragement and love during the writing phases of this book. 